CLINICAL STUDY

\title{
Serum glucocorticoids and adiponectin associate with insulin resistance in children born small for gestational age
}

\author{
S Tenhola ${ }^{1,2}$, B Todorova ${ }^{1}$, J Jääskeläinen ${ }^{1}$, O A Jänne ${ }^{3}$, T Raivio ${ }^{3,4}$ and R Voutilainen ${ }^{1}$ \\ ${ }^{1}$ Department of Pediatrics, University of Kuopio and Kuopio University Hospital, PO Box 1777, FI-70211 Kuopio, Finland, ${ }^{2}$ Department of Pediatrics, \\ Kymenlaakso Central Hospital, Kotkantie 41, FI-48211 Kotka, Finland, ${ }^{3}$ Biomedicum Helsinki, Institute of Biomedicine/Physiology, University of \\ Helsinki, FI-O0014 Helsinki, Finland and ${ }^{4}$ Hospital for Children and Adolescents, University of Helsinki and Helsinki University Central Hospital, \\ FI-00014 Helsinki, Finland
}

(Correspondence should be addressed to R Voutilainen; Email: raimo.voutilainen@uef.fi)

\begin{abstract}
Objectives: Altered glucocorticoid activity is one possible mechanism linking fetal growth restriction with later insulin resistance (IR) and type 2 diabetes. We aimed to investigate whether serum glucocorticoid parameters are related to IR in children born small for gestational age (SGA).

Design: A total of 110 children (55 age- and gender-matched pairs born SGA or appropriate for gestational age (AGA) in a case-control setting) were studied at the mean age of 12.2 (s.D. 0.2) years. Methods: Serum cortisol, corticosteroid-binding globulin (CBG), free cortisol index (FCI = cortisol/CBG), and glucocorticoid bioactivity (GBA, transactivation assay) were analyzed and related to serum adiponectin and insulin-like growth factor-binding protein 1 (IGFBP1) concentrations and homeostasis model assessment for IR (HOMA-IR) and QUICKI indices.

Results: In the pooled study population, GBA correlated well with cortisol and FCI $(r=0.681$ and 0.586 respectively; $P<0.001$ for both). Serum cortisol, CBG, FCI, GBA, HOMA-IR, or QUICKI did not differ between the SGA and AGA subjects, but the SGA children had lower body mass index $(P=0.005)$ and waist circumference (WC) $(P=0.001)$. The mean GBA in the highest GBA quartile was higher among the SGA subjects than among the AGA subjects (138.6 vs $96.4 \mathrm{nmol} / \mathrm{l}$ cortisol equivalents, $P<0.001)$. In the SGA children, GBA correlated positively with HOMA-IR $(r=0.522, P<0.001)$ and inversely with adiponectin $(r=-0.278, P=0.042)(\mathrm{WC} /$ height ratio adjustments), and in logistic regression analysis, higher GBA (odds ratio (OR) 1.3; $P=0.013$ ), lower adiponectin (OR 1.4; $P=0.038)$, and lower IGFBP1 (OR 1.9; $P=0.010)$ associated independently with higher HOMA-IR. Conclusions: These findings suggest that increased glucocorticoid activity and low serum adiponectin concentrations associate with IR in SGA children.
\end{abstract}

European Journal of Endocrinology 162 551-557

\section{Introduction}

The association between growth restriction in early life and cardiovascular morbidity in adulthood was first reported by Barker et al. two decades ago (1). However, the mechanisms explaining this association are not yet understood, although altered programming of the hypothalamic-pituitary-adrenal (HPA) axis could be involved.

Previous experimental studies have suggested that fetal overexposure to glucocorticoids of maternal or fetal origin reduces birth weight and leads to programming in utero with persistently increased glucocorticoid action throughout life $(2,3)$. In addition, mild protein restriction during pregnancy has been reported to reduce $11 \beta$-hydroxysteroid dehydrogenase type 2 enzyme activity in offspring's kidneys and cause persistently increased blood pressure (4).
Thus, increased glucocorticoid activity could contribute to the long-term consequences of early growth restriction.

In humans, low birth weight has been associated with increased plasma cortisol concentrations (5-7), enhanced cortisol response to ACTH stimulation, and features of metabolic syndrome in adulthood (8-10). In children, Clark et al. reported an association between low birth weight and increased urinary glucocorticoid metabolite excretion (11), while other investigators have not been able to show any association between birth size and circulating cortisol concentrations (12-16). Thus, according to these studies, the association between fetal growth restriction and later increased glucocorticoid activity in humans is still controversial, at least during childhood.

Our aims were to investigate whether circulating glucocorticoid bioactivity (GBA), measured by a 
transactivation assay (17), differs between children born small for gestational age (SGA) and appropriate for gestational age (AGA), and whether GBA is related to insulin resistance (IR) and serum adiponectin in SGA children. Serum adiponectin concentrations have been reported to decrease in obesity and insulin-resistant states (reviewed in (18)).

\section{Subjects and methods}

\section{Definitions}

SGA was defined as birth weight or length or ponderal index $>2$ s.D. scores below the respective mean for the gestational age and sex (19). The ponderal index was calculated as (weight $(\mathrm{g}) /$ length $\left.^{3}(\mathrm{~cm})\right) \times 100$. AGA was defined as birth weight, birth length, and ponderal index $\geq-2$ s.D. scores and $\leq 2$ s.D. scores of the respective mean for the gestational age and sex. Homeostasis model assessment for IR (HOMA)-IR index was calculated as $(($ fasting insulin, $\mu \mathrm{U} / \mathrm{ml}) \times($ fasting glucose, $\mathrm{mmol} / \mathrm{l})) / 22.5$ and QUICKI as $1 /(\log$ (fasting insulin, $\mu \mathrm{U} / \mathrm{ml})+\log$ (fasting glucose, mg/dl)) (see (20)). Free cortisol index (FCI) was calculated as serum cortisol (nmol/l)/corticosteroidbinding globulin (CBG) (mg/l) (21).

\section{Subjects}

The study population consisted of all full-term children who were born SGA at Kuopio University Hospital, Finland, between 1984 and 1986; 73 SGA children (70 singletons and three twins) were included in the study. Each SGA child had the next born full-term AGA child matched for sex as the control subject. At the age of 12 years, 55 SGA children (20 boys and 35 girls) and
55 AGA control subjects participated in this study. Nine mothers $(16 \%)$ of the SGA subjects were diagnosed to have arterial hypertension (measured blood pressure more than $140 / 90 \mathrm{mmHg}$ at least two times) during pregnancy; five of these $(9.1 \%$ of the SGA mothers) had preeclampsia. None of the SGA subjects had chromosomal abnormalities, recognizable genetic diseases, or congenital anomalies. The mean age in both the SGA and AGA groups was 12.2 (s.D. 0.2) years. The study protocol was approved by the Research Ethics Committee of Kuopio University Hospital. Informed written consent was obtained from the child and the parents.

\section{Anthropometric characteristics}

Anthropometric data of the study subjects at birth and at 12 years of age are given in Table 1; they have been reported in more detail previously (22). The mean (s.D.) gestational age was 39.0 (1.4) weeks in the SGA group and 39.7 (1.5) weeks in the AGA group. The mean birth measures of the SGA children who dropped out of the study did not differ from those of the participating SGA children.

\section{Blood sampling and laboratory analyses}

Blood samples were taken in the morning, between 0900 and $1000 \mathrm{~h}$, after an overnight fast. An i.v. cannula was placed in the antecubital vein for blood sampling, and blood samples were drawn through the cannula after the child had rested for $1 \mathrm{~h}$. Blood glucose concentrations were determined by a glucose oxidase method (enzyme electrode, Nova Biomedical, Waltham, MA, USA). Serum insulin levels were determined by RIA (Phadeseph Insulin RIA, Pharmacia \& Upjohn, Inc.,

Table 1 Anthropometric characteristics and parameters of glucocorticoid and insulin metabolism in 12-year-old children born full-term and small (SGA) or appropriate (AGA) for gestational age. Means (s.D.) are shown.

\begin{tabular}{|c|c|c|c|}
\hline Variable & $\begin{array}{c}\text { SGA }(n=55) \\
\text { (35 females and } 20 \text { males) }\end{array}$ & $\begin{array}{c}\text { AGA }(n=55) \\
\text { (35 females and } 20 \text { males) }\end{array}$ & $P$ value $^{\mathrm{a}}$ \\
\hline Birth weight (g) & $2452(314)$ & $3455(475)$ & $<0.001$ \\
\hline Birth weight (SDS) & $-2.44(0.53)$ & $-0.24(0.99)$ & $<0.001$ \\
\hline Birth length $(\mathrm{cm})$ & $46.2(2.1)$ & $50.4(2.3)$ & $<0.001$ \\
\hline Birth length (SDS) & $-2.27(0.95)$ & $-0.08(1.16)$ & $<0.001$ \\
\hline Height 12 years (SDS) & $-0.16(0.90)$ & $0.60(1.0)$ & $<0.001$ \\
\hline Weight for height 12 years (\%) & 99 (17) & 109 (22) & 0.021 \\
\hline BMI 12 years $\left(\mathrm{kg} / \mathrm{m}^{2}\right)$ & $17.5(2.9)$ & $19.8(4.2)$ & 0.005 \\
\hline Waist circumference 12 years $(\mathrm{cm})$ & $62.5(7.1)$ & $69.6(11.1)$ & 0.001 \\
\hline Waist circumference/height 12 years & $0.42(0.05)$ & $0.45(0.06)$ & 0.013 \\
\hline S-GBA (nmol/l cortisol equivalents) & $78.0(44.6)$ & $64.9(22.9)$ & 0.127 \\
\hline S-cortisol $(\mathrm{nmol} / \mathrm{l})$ & $292.5(137.4)$ & $272.1(92.8)$ & 0.624 \\
\hline S-CBG (mg/l) & $64.0(13.9)$ & $65.3(14.1)$ & 0.795 \\
\hline Free cortisol index (nmol/mg) & $4.68(2.19)$ & $4.27(1.48)$ & 0.456 \\
\hline HOMA-IR index & $1.91(0.74)$ & $2.03(0.96)$ & 0.763 \\
\hline QUICKI & $0.35(0.02)$ & $0.35(0.02)$ & 0.987 \\
\hline S-adiponectin (mg/l) & $10.3(5.5)$ & $11.1(6.7)$ & 0.388 \\
\hline S-IGFBP1 $(\mu \mathrm{g} / \mathrm{l})$ & $70.0(34.8)$ & $58.8(30.3)$ & 0.090 \\
\hline
\end{tabular}

aWilcoxon matched-pairs signed rank test for differences. Pubertal development stages (no difference between the study groups), S-cortisol concentrations (14) and IGFBP1 data (31) have been reported previously. 
Uppsala, Sweden). Serum adiponectin and insulin-like growth factor-binding protein 1 (IGFBP1) concentrations were measured by ELISA (Quantikine DRP300, R\&D Systems Inc., Minneapolis, MN, USA and DSL-10-7800 ACTIVE Total IGFBP1 ELISA, Diagnostic Systems Laboratories, Inc., Webster, TX, USA, respectively). Serum cortisol was analyzed by the DPC Immulite 2000 chemiluminescent enzyme immunoassay (Diagnostic Products Corporation, Los Angeles, CA, USA). Serum CBG was measured by RIA (CBG-RIA-100, Biosource Europe, Nivelles, Belgium). GBA was measured directly from $10-\mu$ l serum samples using a recombinant cell bioassay in which COS-1 cells are transfected with expression vectors encoding the human glucocorticoid receptor and the nuclear receptor coregulator androgen receptor-interacting protein 3, together with an appropriate reporter gene (luciferase), as described previously (17).

\section{Statistical analyses}

Data were analyzed using the statistical program SPSS for Windows, release 16.0 (SPSS, Inc., Chicago, IL, USA). All continuous variables were examined for normality with the Kolmogorov-Smirnov test. Because the majority of variables distributed nonnormally, the Wilcoxon matched-pairs signed rank test was used for SGA-AGA comparisons and the Mann-Whitney test was used for subgroup comparisons. Frequencies of categorical variables between the study groups were compared with the $\chi^{2}$ test. Correlation coefficients were examined by either Spearman's or Pearson's correlation test. Multiple logistic regression analysis was used to analyze the factors associating with HOMA-IR index. A significance level of $P<0.05$ was used for all analyses.

\section{Results}

\section{Parameters of glucocorticoid and insulin metabolism in SGA and AGA subjects}

In the combined SGA and AGA groups, serum GBA values correlated strongly with serum cortisol concentrations measured by the immunoassay $(r=0.681$, $P<0.001)$ as well as with the FCI derived from the cortisol/CBG ratio $(r=0.586, P<0.001)$. The means of serum GBA, cortisol, CBG, or FCI did not differ between the SGA and AGA children (Table 1), but the distributions of GBA, cortisol, and FCI were skewed to high values in the SGA group (demonstrated by higher S.D. values in the SGA group than in the AGA group, Table 1). Consequently, the mean GBA in the highest GBA quartile was significantly higher among the SGA subjects than among the AGA subjects (138.6 vs $96.4 \mathrm{nmol} / \mathrm{l}$ cortisol equivalents, $P<0.001)$. No significant differences were found in the means of HOMA-IR or QUICKI index between the SGA and AGA groups.
Neither the mean serum IGFBP1 nor adiponectin concentrations differed between the two groups (Table 1). The means of adiponectin, cortisol, FCI, or GBA did not differ between the SGA subgroups with good and poor catch-up growth (detailed data not shown). It is worth noting that the SGA subjects were shorter, lighter, and slimmer (in terms of weight for height, body mass index (BMI), waist circumference (WC), and WC/height ratio) than the AGA controls (Table 1), but their pubertal development stage did not differ from that of the controls at the time of evaluation (14).

\section{The relationship between serum GBA with HOMA-IR and QUICKI indices and serum adiponectin levels}

In the SGA group, serum GBA correlated significantly with HOMA-IR and QUICKI indices $(r=0.410$, $P=0.002$ and $r=-0.410, P=0.002)$. The correlation between GBA and HOMA-IR index was even stronger when adjusted for $\mathrm{WC} /$ height ratio (reflecting the relative amount of visceral fat; $r=0.522, P<0.001$; Fig. 1). Moreover, in the SGA subjects, FCI correlated significantly with HOMA-IR and QUICKI indices, but only when adjusted for BMI $(r=0.356, P=0.008$ and $r=-0.313, P=0.021$ respectively) or $\mathrm{WC} /$ height ratio $(r=0.338, P=0.012$ and $r=-0.298, \quad P=0.029$ respectively). In the SGA children, serum GBA correlated inversely with serum adiponectin $(r=-0.271$, $P=0.045$ and $r=-0.278, P=0.042$ when adjusted for $\mathrm{WC} /$ height ratio), and serum adiponectin correlated inversely with HOMA-IR and positively with QUICKI $(r=-0.443, P=0.001$ and $r=0.443, \quad P=0.001$

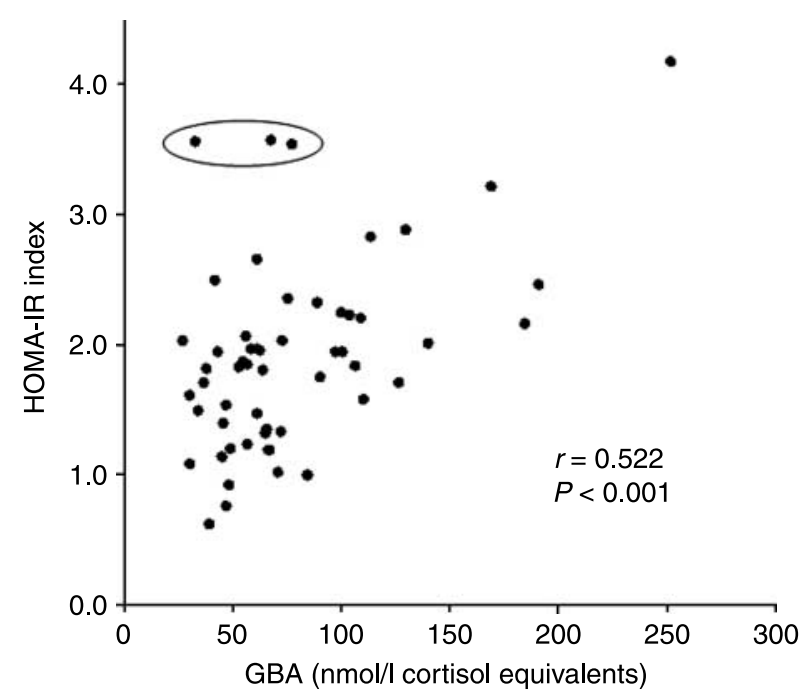

Figure 1 Correlation between HOMA-IR index and glucocorticoid bioactivity (GBA) in the SGA children. The $r$ and $P$ values in the figure are those adjusted for waist circumference (WC)/height ratio (reflecting the amount of visceral fat). The three outliers (circled in the figure) were pubertal girls (Tanner breast stages 3-4) with their weights for height $106-122 \%$ and WC/height ratios $0.43-0.46$ (both above the mean of the whole SGA group). 
Table 2 Parameters of insulin and glucocorticoid metabolism and 24-h systolic ambulatory blood pressure, body mass index (BMI), and waist circumference values in the highest and the lower homeostasis model assessment for insulin resistance (HOMA-IR) quartiles among the 12-year-old children born full-term and small for gestational age (SGA). Means (S.D.) are shown.

\begin{tabular}{|c|c|c|c|}
\hline Variable & $\begin{array}{c}\text { The highest HOMA-IR quartile } \\
\text { in the SGA group } \\
\text { (n=14; } 4 \text { males and } 10 \text { females) }\end{array}$ & $\begin{array}{c}\text { The lower HOMA-IR quartiles } \\
\text { in the SGA group } \\
(n=41 ; 16 \text { males and } 25 \text { females) }\end{array}$ & $P$ value \\
\hline HOMA-IR index & $2.89(0.62)$ & $1.58(0.41)$ & $<0.001$ \\
\hline QUICKI & $0.33(0.01)$ & $0.36(0.02)$ & $<0.001$ \\
\hline S-adiponectin (mg/l) & $7.2(3.8)$ & $11.4(5.6)$ & 0.002 \\
\hline S-IGFBP1 $(\mu \mathrm{g} / \mathrm{l})$ & $44.4(16.9)$ & $78.7(35.2)$ & 0.001 \\
\hline S-GBA (nmol/l cortisol equivalents) & $107.7(60.9)$ & $67.9(32.7)$ & 0.010 \\
\hline S-cortisol (nmol/l) & $360.5(177.1)$ & $269.2(114.5)$ & 0.061 \\
\hline S-CBG (mg/l) & $63.7(14.7)$ & $64.1(13.7)$ & 0.772 \\
\hline Free cortisol index $(\mathrm{nmol} / \mathrm{mg})$ & $5.92(3.04)$ & $4.26(1.66)$ & 0.089 \\
\hline 24-h systolic BP (mmHg) & $120.4(7.5)$ & $116.4(9.0)$ & 0.024 \\
\hline BMI $\left(\mathrm{kg} / \mathrm{m}^{2}\right)$ & $18.4(2.8)$ & $17.3(2.9)$ & 0.164 \\
\hline Waist circumference $(\mathrm{cm})$ & $63.6(7.3)$ & $62.1(7.1)$ & 0.434 \\
\hline Waist circumference/height & $0.43(0.05)$ & $0.41(0.05)$ & 0.306 \\
\hline
\end{tabular}

${ }^{\mathrm{a}}$ The Mann-Whitney test for differences. Sex distribution and pubertal development according to the Tanner B/G staging did not differ between the HOMA-IR subgroups ( $P=0.483$ and 0.218 respectively; $\chi^{2}$ test). The ambulatory blood pressure values of the whole study population have been reported previously (32).

respectively). Furthermore, an inverse correlation was found between serum adiponectin and WC $(r=-0.288, P=0.033)$, and between serum adiponectin and $\mathrm{WC} /$ height ratio $(r=-0.298, P=0.027)$ in the SGA subjects. All these correlations were also examined in the AGA group, and none of them reached statistical significance (data not shown).

\section{Factors associating with HOMA-IR index in the SGA children}

The SGA subjects in the highest HOMA-IR quartile had significantly higher GBA, lower adiponectin and IGFBP1 concentrations, and higher 24-h systolic blood pressure than the SGA subjects in the lower HOMA-IR quartiles. Interestingly, serum cortisol concentrations (measured by the immunoassay) or FCI did not differ significantly between the high and lower HOMA-IR SGA subgroups (Table 2). When similar comparisons were performed in the control group, the AGA subjects in their highest HOMAIR quartile had lower mean IGFBP1 (35.5 vs $65.8 \mu \mathrm{g} / \mathrm{l}, \quad P=0.001)$ and higher BMI (23.9 vs $\left.18.4 \mathrm{~kg} / \mathrm{m}^{2}, P<0.001\right)$ and $\mathrm{WC} /$ height ratio $(0.50$ vs $0.43, P=0.001)$, but adiponectin $(P=0.334)$, GBA $(P=0.440)$, and other glucocorticoid parameters or blood pressure levels did not differ when compared to those of the AGA subjects in the lower HOMA-IR quartiles (detailed data not shown). For the multiple logistic regression analysis, the dependent variable HOMA-IR was dichotomized using the 75 th percentile level as the cut-off point. In this analysis, higher serum GBA and lower adiponectin and IGFBP1 levels associated independently with higher HOMA-IR index in the SGA group (Table 3). An increase of $10 \mathrm{nmol} / \mathrm{l}$ cortisol equivalents in serum GBA increased by 1.3 -fold the risk of higher HOMA-IR, while a decrease of $1 \mathrm{mg} / \mathrm{l}$ in serum adiponectin associated with a 1.4-fold and a decrease of $10 \mu \mathrm{g} / \mathrm{l}$ in serum IGFBP1 with a 1.9-fold risk of high HOMA-IR. The model was adjusted for WC/height ratio, sex, and pubertal stage, none of which was independently associated with high HOMA-IR. The model used explained $61.1 \%$ of the variation of HOMA-IR (Table 3 ).

\section{Discussion}

The present cohort study showed no differences in the mean serum GBA levels or other glucocorticoid parameters between the SGA children and their sexand age-matched AGA controls. However, in the SGA group, serum GBA values exceeding the 75 th percentile were significantly higher than those in the AGA group. Furthermore, the SGA subjects with the highest

Table 3 Factors predicting high homeostasis model assessment for insulin resistance (HOMA-IR) index in the small for gestational age children. Multiple logistic regression analysis $(n=55)^{\mathrm{a}}$.

\begin{tabular}{lcccc}
\hline Covariate & Regression coefficient & Significance & Odds ratio & Confidence interval (95\%) \\
\hline High GBA level $(10 \mathrm{nmol} / \mathrm{I}$ & 0.27 & 0.013 & 1.3 & $1.1-1.6$ \\
$\quad$ cortisol equivalents) & 0.37 & 0.038 & 1.4 & $1.0-2.0$ \\
Low adiponectin level $(\mathrm{mg} / \mathrm{l})$ & 0.67 & 0.010 & 1.9 & $1.2-3.1$ \\
Low IGFBP1 $(10 \mu \mathrm{g} / \mathrm{l})$ & -0.05 & 0.657 & 1.0 & $0.8-1.2$ \\
Waist circumference/height $(\%)$ & & & \\
\hline
\end{tabular}

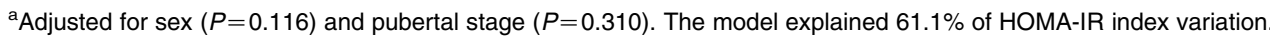


HOMA-IR index had higher serum GBA and lower adiponectin and IGFBP1 levels than those with lower HOMA-IR index. These findings suggest that there is an SGA subgroup with a more insulin-resistant state detectable already in childhood, and that increased glucocorticoid activity associates with it.

Previous studies have revealed increased plasma cortisol concentrations or their reactivity, IR, and type 2 diabetes in adult subjects born with low birth weight $(5,6,10,11)$. Cortisol excess in humans, e.g. in Cushing's syndrome or during pharmacologic glucocorticoid treatment, may induce adverse effects including the components of the metabolic syndrome (hypertension, IR, dyslipidemia, and visceral obesity) (reviewed in (23)). Reynolds et al. (10) reported that men with low birth weight had enhanced responses of plasma cortisol to ACTH and increased total urinary cortisol metabolite excretion. In these subjects, features of metabolic syndrome such as raised blood pressure, glucose intolerance, and hypertriglyceridemia were associated with enhanced adrenal responsiveness to ACTH (10). The present study showed an association between GBA, measured by the transactivation assay, and IR indices in SGA children. Accordingly, intrauterine growth restriction may lead to alterations in glucocorticoid activity, which may contribute to IR.

Serum GBA associated more clearly than immunologically measured cortisol concentrations or FCI with HOMA-IR index in the SGA children. More than $90 \%$ of circulating cortisol in human serum is bound to proteins (CBG and albumin). FCI, calculated as a ratio of serum total cortisol to $\mathrm{CBG}$, eliminates the effect of CBG variation in total cortisol values, and FCI has thus been shown to correlate with free serum cortisol, which is considered the biologically active hormone (21). Calculation of FCI does not eliminate the influence of variable albumin concentrations on total cortisol levels. We did not measure serum albumin concentration in our study, but we could speculate that the SGA subjects might have had slightly lower serum albumin levels as they were quite slim and their mean BMI was significantly lower than that of the AGA controls (Table 1, (22)). At least theoretically, variation in serum albumin concentrations could explain the better association of GBA than that of immunologically measured cortisol concentrations or FCI with HOMA-IR index in our study. We assume that our GBA measurement, based on a transactivation assay, estimates the circulating bioavailable cortisol concentrations better than the total cortisol measurement or even the calculated FCI. Thus, GBA measurements can give useful additional information (compared to immunological cortisol assays) on the endogenous activity of the HPA axis and cortisol metabolism. Apparently, GBA measurements have even greater value in conditions where the combined effects of exogenous synthetic glucocorticoids and endogenous cortisol on circulating glucocorticoid milieu are evaluated (24).
Adiponectin is an adipocyte-specific protein with insulin-sensitizing, anti-atherogenic, and antiinflammatory properties. Circulating adiponectin is decreased in obesity, IR, and type 2 diabetes (reviewed in (18)). In our study, low adiponectin levels associated with higher HOMA-IR and lower QUICKI indices, i.e. decreased insulin sensitivity, in the SGA children. In addition, serum adiponectin showed a negative correlation with WC and WC/height ratio, suggesting that some SGA children might have extra visceral fat explaining reduced adiponectin levels even though they were not overweight or obese. This type of association was recently reported in somewhat younger SGA children (25). Glucocorticoids have a negative effect on adiponectin $(26,27)$. Dexamethasone inhibited adiponectin release from human adipocytes (26). Furthermore, decreased circulating adiponectin levels were found in nonobese patients with Cushing's syndrome and in healthy subjects after i.v. hydrocortisone administration (27). Cianfarani et al. reported that SGA children had lower adiponectin levels than their AGA control children (28), and that the adiponectin levels were even lower in those SGA children with good postnatal catch-up growth than in those without it $(28,29)$. In contrast, Evagelidou et al. (30) found that adiponectin levels had a trend to be slightly higher in SGA children than in their control children. The present study could not find any difference in adiponectin concentrations between SGA and AGA children, and no difference in adiponectin levels was found between the SGA children with good or poor catch-up growth. However, in our SGA subjects, serum adiponectin concentrations correlated inversely with GBA levels even when adjusted for $\mathrm{WC} /$ height ratio, which suggests an independent association between GBA and adiponectin. This finding is consistent with the previous reports on the relationship between adiponectin and glucocorticoids $(26,27)$.

The reasons for being born SGA are numerous and include fetal, uteroplacental, and maternal factors. The possible mechanisms explaining increased cardiovascular morbidity in adults born SGA (Barker's hypothesis) may also vary depending on the etiology of the intrauterine growth restriction. Thus, it is not surprising that it has been difficult to find conclusive evidence for a single mechanism explaining the association between small birth size and cardiovascular morbidity in adulthood. The current study showed that the subgroup of SGA children with the highest HOMAIR index had significantly higher GBA than the SGA subjects with lower HOMA-IR index, and that GBA turned out to associate independently of obesity (BMI or WC/height ratio) with high HOMA-IR index. Low serum adiponectin level also associated independently of obesity with high HOMA-IR index. Furthermore, high GBA levels associated independently of obesity with low adiponectin levels. Thus, increased 
glucocorticoid activity may increase IR in SGA subjects by several mechanisms.

The strengths of this study were the carefully matched SGA and AGA children with a similar age at examination. Moreover, the blood samples were taken exactly between 0900 and $1000 \mathrm{~h}$, minimizing the effect of diurnal variation on both cortisol and adiponectin concentrations. The transactivation-based GBA assay turned out to give additional information compared with the traditional cortisol assay and FCI. Obviously, it is desirable that our findings will be replicated in other (hopefully larger) studies.

In conclusion, there is an SGA subgroup with a more insulin-resistant state detectable already in childhood. Increased glucocorticoid activity and low adiponectin associate independently with this increased IR. GBA measurement, based on a transactivation assay, seems to estimate the circulating bioavailable cortisol concentrations better than the total cortisol measurement or the calculated FCI.

\section{Declaration of interest}

The authors declare that there is no conflict of interest that could be perceived as prejudicing the impartiality of the research reported.

\section{Funding}

This work was supported by Sigrid Jusélius Foundation and Kuopio University Hospital.

\section{Acknowledgements}

Minna Heiskanen and Leila Antikainen are thanked for their skillful technical assistance.

\section{References}

1 Barker DJP, Winter PD, Osmond C, Margetts B \& Simmonds SJ. Weight in infancy and death from ischaemic heart disease. Lancet 19892 577-580.

2 Benediktsson R, Lindsay RS, Noble J, Seckl JR \& Edwards CR. Glucocorticoid exposure in utero: a new model for adult hypertension. Lancet $1993 \mathbf{3 4 1} 339-341$.

3 Seckl JR. Prenatal glucocorticoids and long-term programming. European Journal of Endocrinology 2004151 49-62.

4 Bertram C, Trowern AR, Copin N, Jackson AA \& Whorwood CB. The maternal diet during pregnancy programs altered expression of the glucocorticoid receptor and type $211 \beta$-hydroxysteroid dehydrogenase: potential molecular mechanisms underlying the programming of hypertension in utero. Endocrinology 2001142 2841-2853.

5 Phillips DIW, Barker DJP, Fall CHD, Seckl JR, Whorwood CB, Wood P \& Walker BR. Elevated plasma cortisol concentrations: a link between low birth weight and the insulin resistance syndrome? Journal of Clinical Endocrinology and Metabolism 1998 83 757-760.

6 Phillips DIW, Walker BR, Reynolds RM, Flanagan DEH, Wood PJ, Osmond C, Barker DJP \& Whorwood CB. Low birth weight predicts elevated plasma cortisol concentrations in adults from 3 populations. Hypertension 200035 1301-1306.
7 van Montfoort N, Finken MJJ, le Cessie S, Dekker FW \& Wit JM. Could cortisol explain the association between birth weight and cardiovascular diseases in later life? A meta-analysis European Journal of Endocrinology 2005153 811-817.

8 Levitt NS, Lambert EV, Woods D, Hales N, Andrew R \& Seckl JR. Impaired glucose tolerance and elevated blood pressure in low birth weight, nonobese, young South African adults: early programming of cortisol axis. Journal of Clinical Endocrinology and Metabolism 200085 4611-4618.

9 Reynolds RM, Walker BR, Syddall HE, Andrew R, Wood PJ, Whorwood CB \& Phillips DIW. Altered control of cortisol secretion in adult men with low birth weight and cardiovascular risk factors. Journal of Clinical Endocrinology and Metabolism 200186 245-250.

10 Reynolds RM, Walker BR, Syddall HE, Andrew R, Wood P \& Phillips DIW. Is there a gender difference in the associations of birth weight and adult hypothalamic-pituitary-adrenal axis activity? European Journal of Endocrinology 2005152 249-253.

11 Clark PM, Hindmarsh PC, Shiell AW, Law CM, Honour JW \& Barker DJP. Size at birth and adrenocortical function in childhood. Clinical Endocrinology 199645 721-726.

12 Dahlgren J, Boguszewski M, Rosberg S \& Albertsson-Wikland K. Adrenal steroid hormones in short children born small for gestational age. Clinical Endocrinology 199849 353-361.

13 Houang M, Morineau G, Le Bouc Y, Fiet J \& Gourmelen M. The cortisol-cortisone shuttle in children born with intrauterine growth retardation. Pediatric Research 199946 189-193.

14 Tenhola S, Martikainen A, Rahiala E, Parviainen M, Halonen P \& Voutilainen R. Increased adrenocortical and adrenomedullary hormonal activity in 12-year-old children born small for gestational age. Journal of Pediatrics 2002141 477-482.

15 Radetti G, Renzuello L, Gottardi E, D’Addato G \& Messner H. Altered thyroid and adrenal function in children born at term and preterm, small for gestational age. Journal of Clinical Endocrinology and Metabolism $2004896320-6324$.

16 Ong KK, Potau N, Petry CJ, Jones R, Ness AR, The Avon Longitudinal Study of Parents and Children (ALSPAC) Study Team, Honour JW, DeZegher F, Ibánez L \& Dunger DB. Opposing influences of prenatal and postnatal weight gain on adrenarche in normal boys and girls. Journal of Clinical Endocrinology and Metabolism 200489 2647-2651.

17 Raivio T, Palvimo JJ, Kannisto S, Voutilainen R \& Jänne OA. Transactivation assay for determination of glucocorticoid bioactivity in human serum. Journal of Clinical Endocrinology and Metabolism 200287 3740-3744.

18 Lihn AS, Pedersen SB \& Richelsen B. Adiponectin: action, regulation and association to insulin sensitivity. Obesity Reviews 20056 13-21.

19 Pihkala J, Hakala T, Voutilainen P \& Raivio K. New Finnish fetal growth charts (in Finnish). Duodecim 1989105 1540-1546.

20 Muniyappa R, Lee S, Chen H \& Quon MJ. Current approaches for assessing insulin sensitivity and resistance in vivo: advantages, limitations, and appropriate usage. American Journal of Physiology. Endocrinology and Metabolism 2008294 E15-E26.

21 Le Roux CW, Sivakumaran S, Alaghband-Zadeh J, Dhillo W, Kong WM \& Wheeler MJ. Free cortisol index as a surrogate marker for serum free cortisol. Annals of Clinical Biochemistry 200239 406-408.

22 Tenhola S, Martikainen A, Rahiala E, Herrgard E, Halonen P \& Voutilainen R. Serum lipid concentrations and growth characteristics in 12-year-old children born small for gestational age. Pediatric Research $2000 \mathbf{4 8} 623-628$.

23 Anagnostis P, Athyros VG, Tziomalos K, Karagiannis A \& Mikhailidis DP. The pathogenetic role of cortisol in the metabolic syndrome: a hypothesis. Journal of Clinical Endocrinology and Metabolism $2009942692-2701$.

24 Nykänen P, Raivio T, Heinonen K, Jänne O \& Voutilainen R. Circulating glucocorticoid bioactivity and serum cortisol concentrations in premature infants: the influence of exogenous glucocorticoids and clinical factors. European Journal of Endocrinology $2007156577-583$. 
25 Ibánez L, Lopez-Bermejo A, Suárez L, Marcos MV, Diaz M \& de Zegher F. Visceral adiposity without overweight in children born small for gestational age. Journal of Clinical Endocrinology and Metabolism $2008932079-2083$.

26 Degawa-Yamauchi M, Moss KA, Bovenkerk JE, Shankar SS, Morrison CL, Lelliott CJ, Vidal-Puig A, Jones RM \& Considine RV. Regulation of adiponectin expression in human adipocytes: effects of adiposity, glucocorticoids, and tumor necrosis factor $\alpha$. Obesity Research 200513 662-669.

27 Fallo F, Scarda A, Sonino N, Paoletta A, Boscaro M, Pagano C, Federspil G \& Vettor R. Effect of glucocorticoids on adiponectin: a study in healthy subjects and in Cushing's syndrome. European Journal of Endocrinology 200450 339-344.

28 Cianfarani S, Martinez C, Maiorana A, Scirè G, Spadoni GL \& Boemi S. Adiponectin levels are reduced in children born small for gestational age and are inversely related to postnatal catch-up growth. Journal of Clinical Endocrinology and Metabolism 200489 1346-1351.

29 Ibánez L, López-Bermejo A, Diaz M, Marcos MV, Casano P \& de Zegher F. Abdominal fat partitioning and high-molecular-weight adiponectin in short children born small for gestational age. Journal of Clinical Endocrinology and Metabolism $2009 \mathbf{9 4}$ 1049-1052.

30 Evagelidou EN, Giapros VI, Challa AS, Kiortsis DN, Tsatsoulis AA \& Andronikou SK. Serum adiponectin levels, insulin resistance, and lipid profile in children born small for gestational age are affected by the severity of growth retardation at birth. European Journal of Endocrinology 2007156 271-277.

31 Tenhola S, Halonen P, Jääskeläinen J \& Voutilainen R. Serum markers of growth hormone and insulin action in 12-year-old children born small for gestational age. European Journal of Endocrinology 2005152 335-340.

32 Rahiala E, Tenhola S, Vanninen E, Herrgård E, Tikanoja T \& Martikainen A. Ambulatory blood pressure in 12-year-old children born small for gestational age. Hypertension 200239 909-913.

Received 17 November 2009

Accepted 16 December 2009 